\title{
PROJ ETO CULTURA E EDUCAÇÃO: UMA NOVA PROPOSTA MUSEOLÓGICA REGIONAL NA DIMENSÃO DO MUSEU HISTÓRICO E GEOGRÁFICO DE POÇOS DE CALDAS MG
}

\author{
Daniel Fernandes Moreira * \\ Haroldo Paes Gessoni ** \\ Sônia Maria Sanches ***
}

\begin{abstract}
Resumo: O Projeto Cultura e Educação: uma nova proposta museológica regional na dimensão do Museu Histórico e Geográfico de Poços de Caldas MG, certificado de aprovação №. 1619/001/2004 foi desenvolvido no ano 2007, através da Lei Estadual de Incentivo a Cultura №. 12.733/97, 13.665/2000 Decreto №. 43.615/2003. É um projeto de caráter artístico-cultural que almeja conscientizar alunos e professores das escolas da rede pública em relação as riquezas pré históricas, históricas, geológicas, arquitetônicas, ecossistêmicas e paisagísticas, através de uma Ação Cultural para professores e confecção de Kits Artísticos Itinerantes.
\end{abstract}

Palavra Chave: Cultura - Pré-História - Geologia - História - Patrimônio.

\section{INTRODUÇÃO}

MOTIVOS QUE LEVARAM A DESENVOLVER O PROJETO

Através do curso de pós-graduação aluno especial ${ }^{1}$ no curso de Arqueologia oferecido no (MAE-USP

\footnotetext{
* Lei Estadual de Incentivo a Cultura do Estado de Minas Gerais. Museu Histórico e Geográfico de Poços de Caldas. E-mail: dafmor78@hotmail.com. (Turismólogo) ** Museu Histórico Geográfico de Poços de Caldas MG. E-mail: hgessoni@uol.com.br (Arquiteto e Urbanista)

*** Museu Histórico e Geográfico de Poços de Caldas MG. E-mail: soniamsanches@gmail.com (Pedagoga) (1) Daniel F Moreira aluno especial no curso de pós graduação em Arqueologia MAE-USP.
}

Museu de Arqueologia e Etnologia da Universidade de São Paulo e como estagiário voluntário no serviço técnico de Musealização desenvolvendo monitorias para o público infantil e adolescentes demonstrando o acervo Formas da Humanidade tivemos contato direto com a metodologia pedagógica dessa instituição a qual realiza plano de treinamento para professores utilizando materiais didáticos como a Valise Pedagógica "Origens do Homem, Kit de Objetos Arqueológicos e Etnográficos, material didático para professores". Com a gama de conhecimentos adquiridos no MAE notamos a necessidade de enfatizar os aspectos pré-históricos, históricos, geoló- 
gicos, arquitetônicos e paisagísticos na cidade de Poços de Caldas, visto que a cidade possui grandes riquezas nas respectivas áreas. Para isso criamos um Kit Pedagógico I tinerante contendo cinco gavetas "Maquetes" que irão ressaltar todos esses aspectos regionais de uma forma lúdica criada através de um trabalho artístico confeccionado por alunos do PMJ (Plano Municipal da Juventude de Poços de Caldas MG). Juntamente com o Kit criamos também um plano de Ação Cultural que será ministrado por um Turismólogo, Arquiteto, Pedagoga.

\section{DESCRI ÇÃO DO PROJ ETO - (Apresentação)}

A proposta do projeto, dividiu-se em duas etapas: A primeira elaborou Três Kits Artísticos Culturais (Maquetes) itinerantes². 0 conteúdo inserido no Kit é o contexto ${ }^{3}$ do potencial do acervo do Museu Histórico e Geográfico.

Os Kits Artísticos Culturais percorrerão os estabelecimentos de ensino das zonas rurais e urbanas como instrumento didático que será criado através de um trabalho “ARTísTI CO" que enriquecerá o currículo escolar do ensino médio e fundamental.

\section{Planta do Kit Artístico Cultural Itinerante}

Primeira Gaveta

\begin{tabular}{|c|}
\hline CIDADE ATUAL \\
DESENVOLV MENTO URBANO \\
\hline Segunda Gaveta \\
$\begin{array}{c}\text { MUSEU HISTÓRI COE GEOGRÁFI CO } \\
\text { DE POÇOS DE CALDAS }\end{array}$ \\
\hline Terceira Gaveta \\
\hline MACI ÇO ALCALI NO \\
DE POÇOS DE CALDAS MG \\
\hline Quarta gaveta \\
\hline FOTOSAI NT HI LAI RE \\
FONTE PEDROBOTEUHO \\
\hline Quinta Gaveta \\
COMPLEXO \\
ARQUI TETÔNICO
\end{tabular}

(2) Os Kits pedagógicos representam maquetes que foram confeccionadas por alunos do PMJ - (Plano Municipal da Juventude de Poços de Caldas). As maquetes possuem cinco gavetas.

(3) 0 contexto refere-se às riquezas naturais e culturais (históricas e pré-históricas) de Poços de Caldas. 
PROJ ETO CULTURA E EDUCAÇÃO: UMA NOVA PROPOSTA MUSEOLÓGICA REGIONAL NA DIMENSÃO DO MUSEU HISTÓRICO E GEOGRÁFICO DE POÇOS DE CALDAS MG Daniel Fernandes Moreira, Haroldo Paes Gessoni e Sônia Maria Sanches

\section{Registro Fotográfico Do Kit Pedagógico}
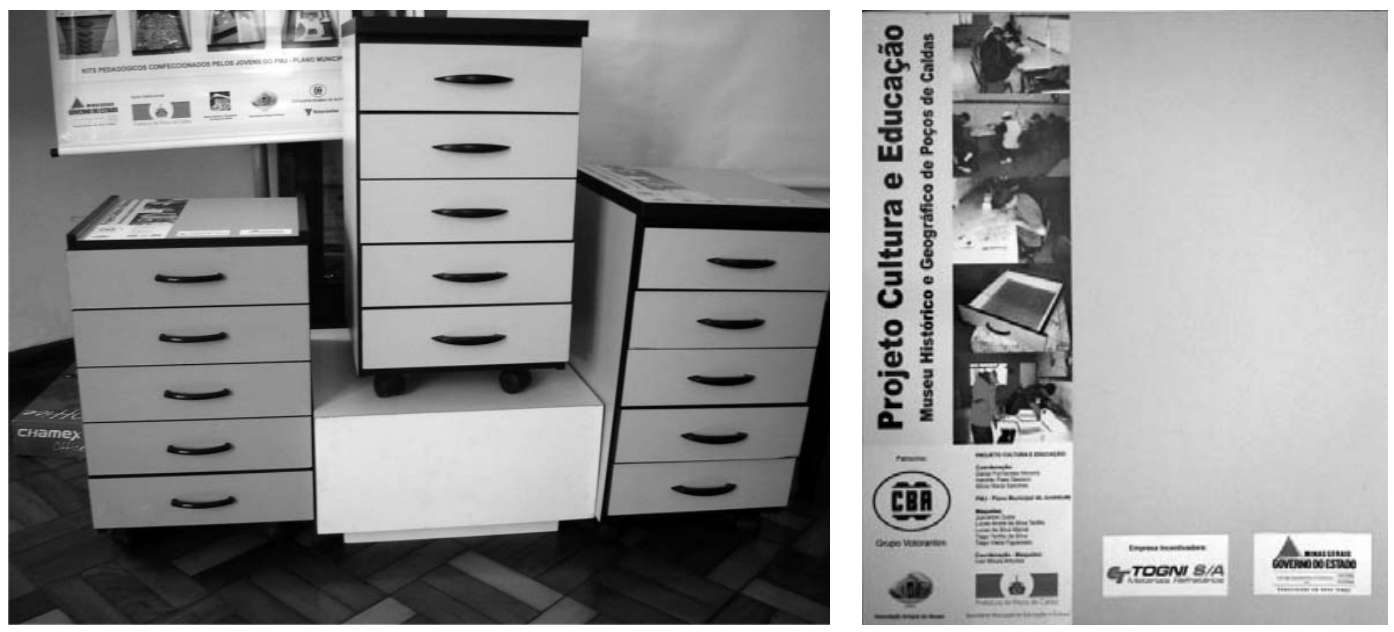

Conteúdo da Gaveta 01 - Representa a cidade de Poços de Caldas atualmente (o núcleo urbano e a Serra de São Domingos).

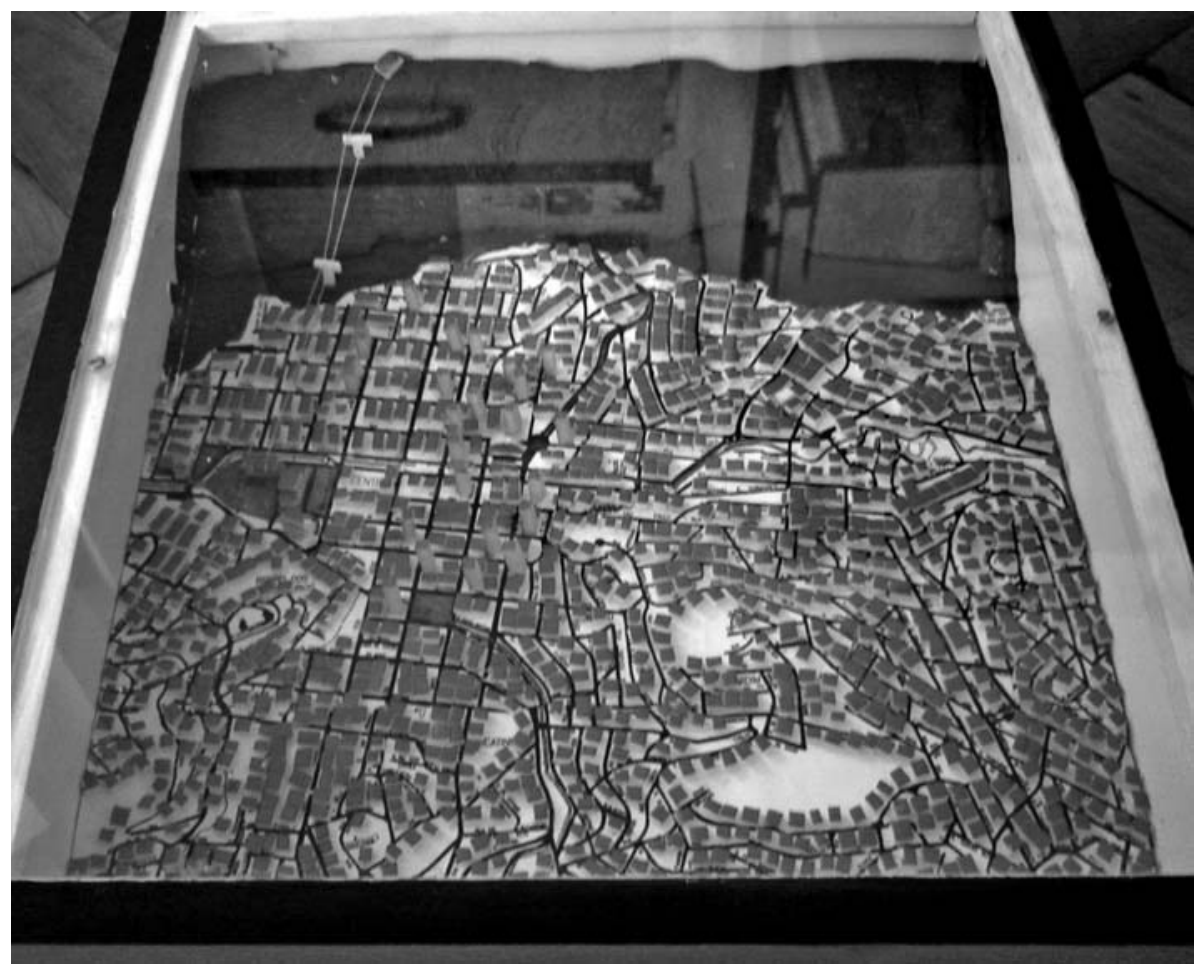




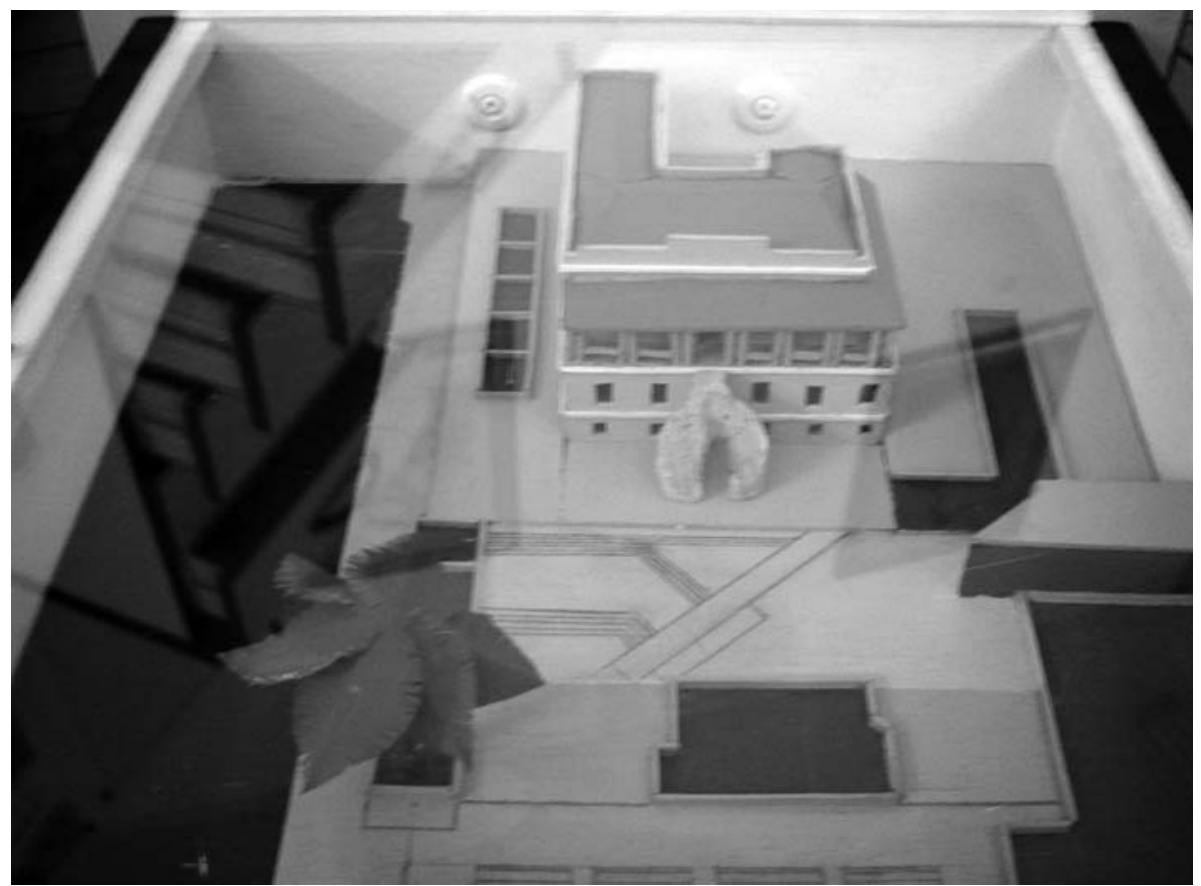

Conteúdo da Gaveta 02 - Representa o museu Histórico e Geográfico de Poços de Caldas MG.

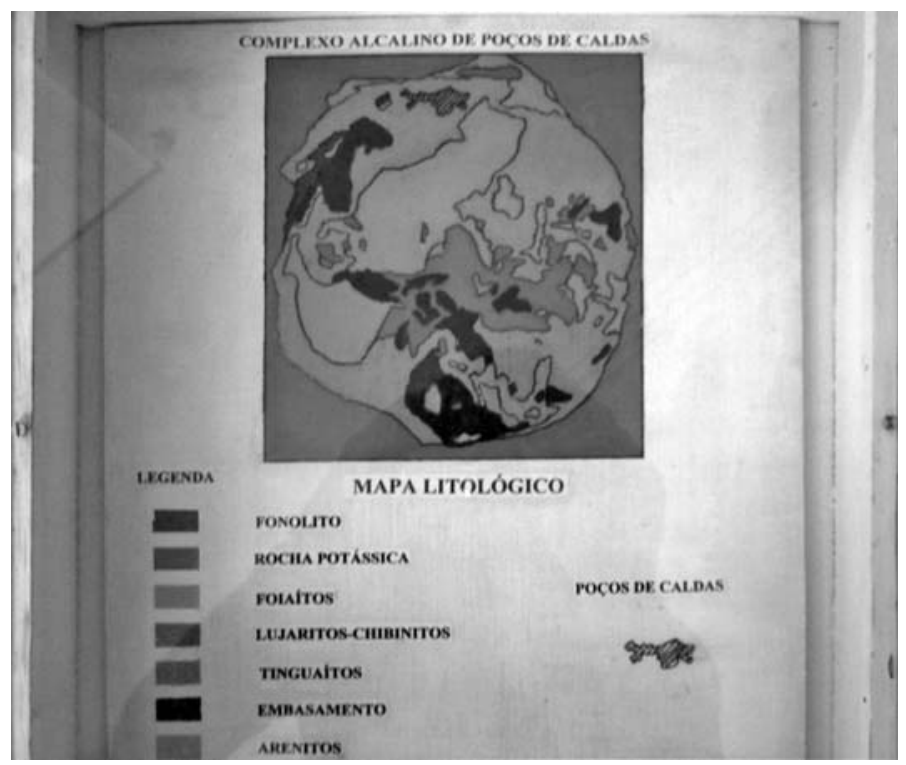

Conteúdo da Gaveta 03 - Representa os diferentes tipos litologicos identificados no Maciço Alcalino de Poços de Caldas MG. 


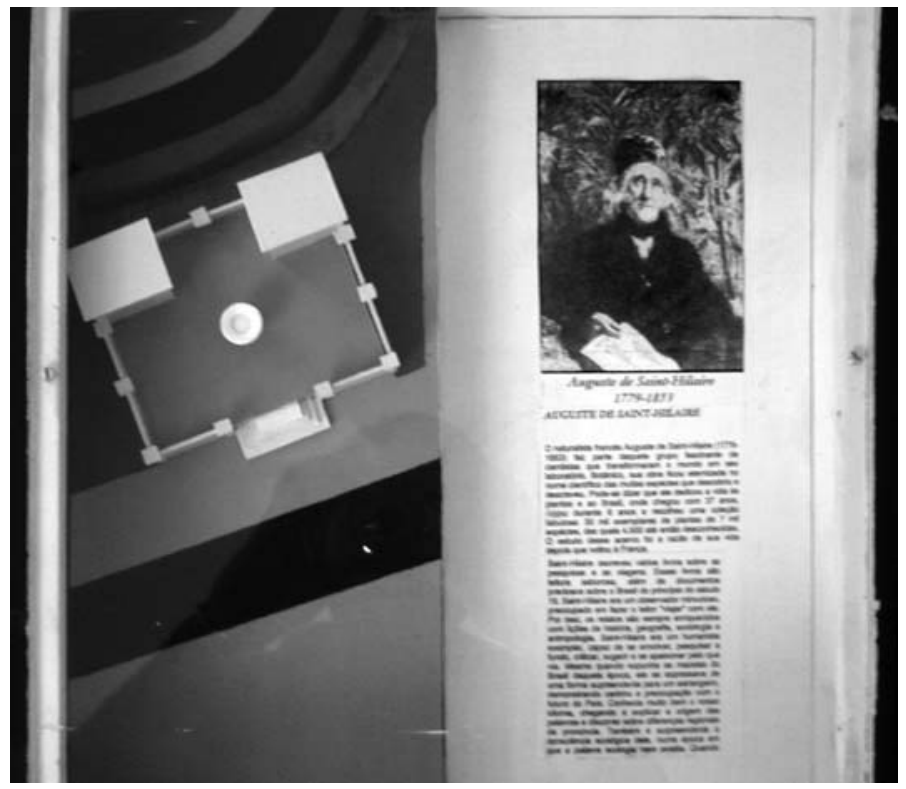

Conteúdo da Quinta gaveta - Representa a primeira Fonte de Agua Termal de Poços de Caldas "Fonte Pedro Botelho (a esquerda) . A direita representamos as descrições de Augusto Saint-Hilare ( naturalista Frances) que relata a existência de águas sulfurosas detectadas em Poços de Caldas MG em 1819.

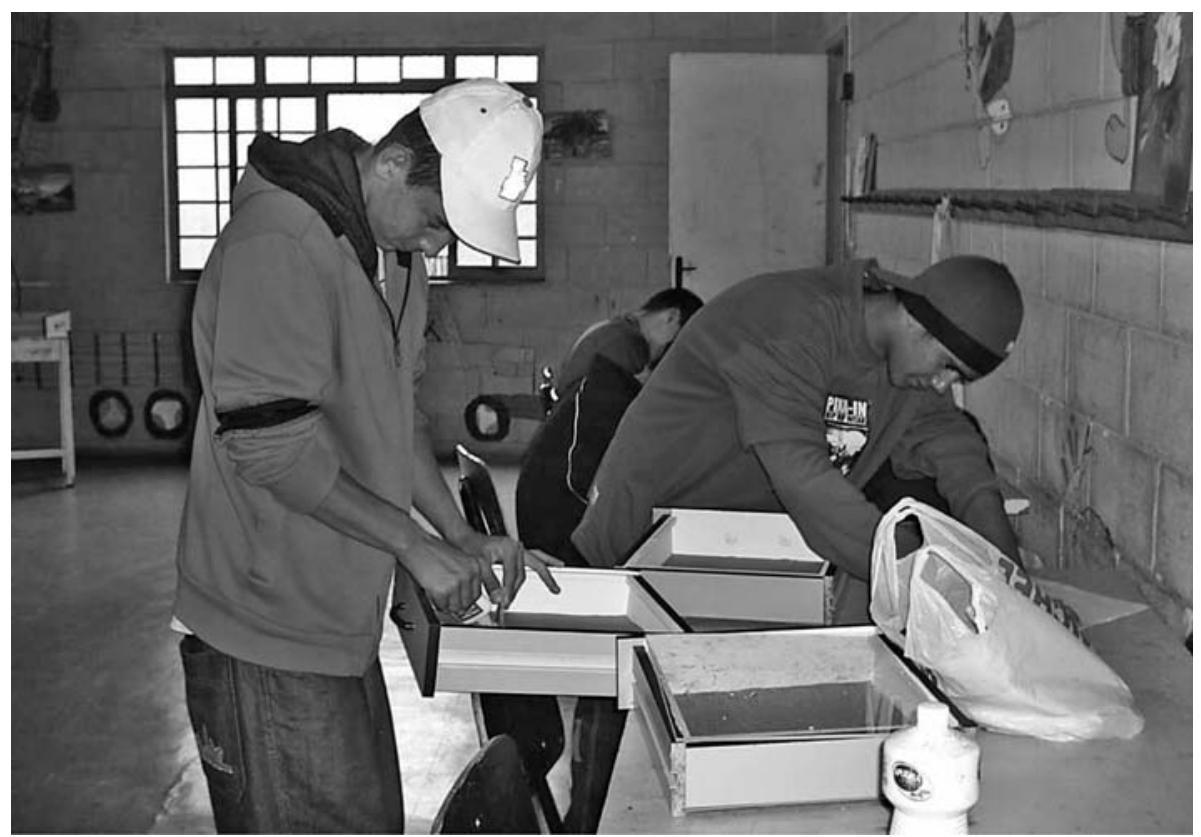

Crianças do Plano Municipal da Juventude (PMJ) confeccionando os Kits sob responsabilidade do coordenador voluntário I van Moura Antunes. 


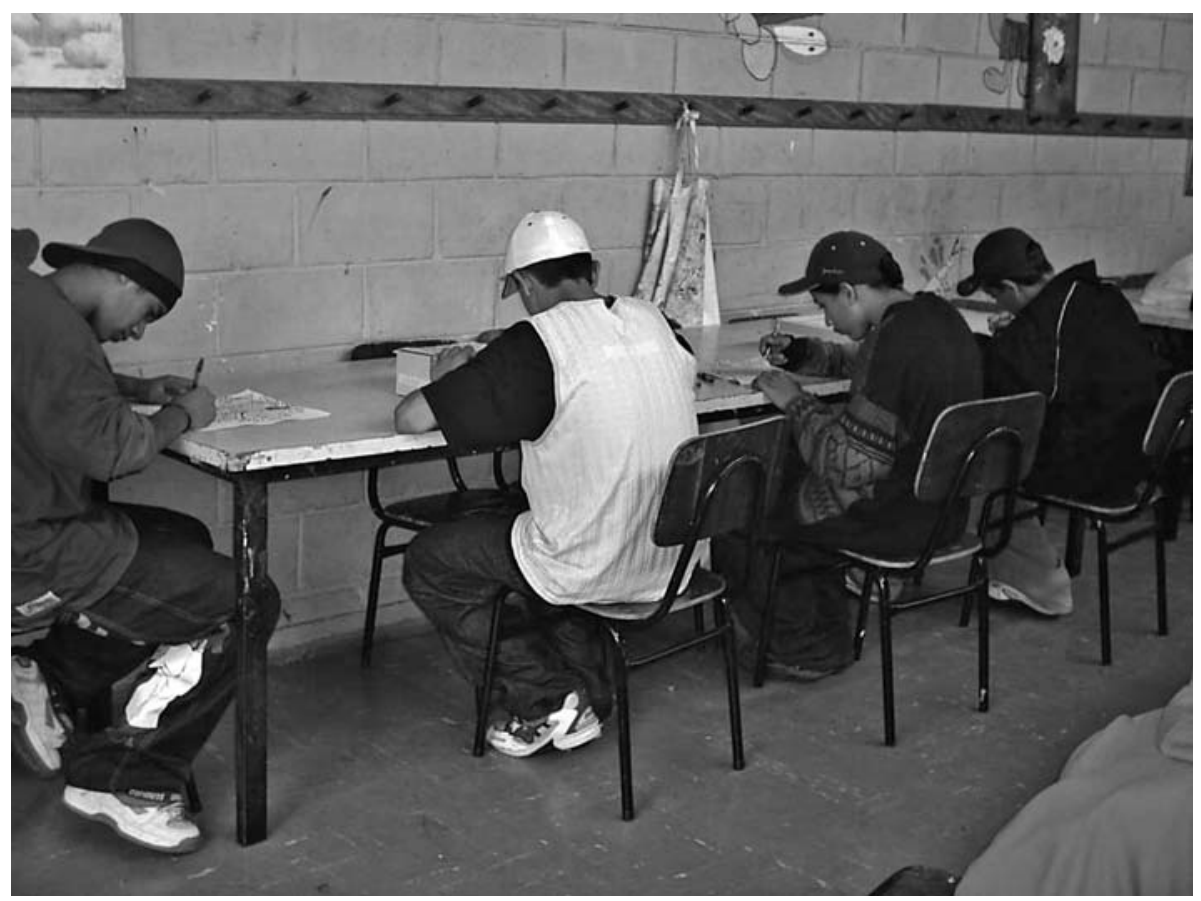

Crianças do Plano Municipal da Juventude (PMJ) confeccionando os Kits sob responsabilidade do coordenador voluntário Ivan Moura Antunes.

Para a segunda etapa, realizamos uma Ação Cultural ${ }^{4}$ destinada para professores da rede pública e possuiu o objetivo de divulgar as riquezas regionais, educando os cidadãos, sensibilizando-os para a necessidade de preservação do patrimônio histórico através do processo de releitura do ambiente natural.

(4) O Plano de Ação Cultural consistiu na confecção de apostilas e aulas expositivas destinadas aos professores de Poços de Caldas, funcionários do Museu e palestras nas empresas que colaboraram com 0 projeto através da Lei Estadual De Incentivo a Cultura do Estado de Minas Gerais (ICMS).

A empresa Incentivadora do projeto foi a Togni S.A(Materiais Refratários), e a empresa colaboradora foi a CBA (Companhia Brasileira de Alumínio-Votorantim. Como suporte e desenvolvimento do projeto enfatizamos a colaboração da AMIV (Associação dos Amigos do Museu), Prefeitura Municipal de Poços de Caldas - MG, Secretaria de Educação de Poços de Caldas MG e Museu Histórico e Geográfico.
Nessa foi realizada a parceria com o Museu Histórico e Geográfico situado na cidade de Poços de Caldas Sul de Minas Gerais.

O projeto inseriu o processo de musealição, pesquisa, comunicação, preservação e arte.

Segue abaixo o plano de Ação Cultural destinado para professores da rede pública, nas zonas rurais e urbanas.

O Plano de Ação Cultural realizou a etapa de divulgação do projeto confeccionando cartazes distribuídos nas escolas, Painel apresentado na IV semana de Museologia oferecido pela Universidade de São Paulo USP- Brasil 2005 e uma publicação no livro Estudos de Arqueologia Histórica/organização de Pedro PauIo Funari e Everson Fogolare, Erechim RS (2005) ISBN 85-905487-1-6.

O projeto realizou a divulgação no Jornal da Mantiqueira, Jornal da Cidade, Jornal Folha Popular, J ornal de Poços, Reportagem na TV Plan, Entrevista na Rádio Difusora. Essas, foram realizadas na data de 29-08-2007. 
Visamos potencializar relações proveitosas entre o museu e o ensino formal.

No final de cada etapa proposta, foram enfatizadas aos grupos de professores participantes várias sugestões didáticas destinadas aos alunos dentro e fora da sala de aula.

\section{Abordagens do Plano de Ação Cultural 01}

- Plano de Ação Cultural

- Primeira Abordagem

- Ministrante: Daniel Fernandes Moreira.

Essa etapa teve como objetivo enfatizar aspectos ligados a evolução do vulcanismo e suas transformações magmáticas ao longo do tempo que originou a formação do maciço Alcalino de Poços de Caldas MG. Procuramos englobar nessa etapa os diferentes tipos litológicos existentes e suas relações com as atividades de intempéries enfatizando o surgimento das águas termais, a ocupação dos grupos pré- históricos e o contato com os colonizadores.

- Abordagens: "Pré-História e História"

- O Maciço Alcalino de Poços de Caldas

- A Geologia do Planalto de Poços de Caldas

- As fissuras, águas termais e mineração.

- Os índios pré-históricos e os vestígios arqueológicos

- O Francês August Saint-Hilare

- Sugestões didáticas

\section{Abordagens do Plano de Ação Cultural 02}

\section{Plano de Ação Cultural}

Segunda Abordagem

Ministrante: Sônia Maria Sanches

Esse treinamento tem como objetivo específico contribuir com o educador (a) do ensino fundamental no sentido de ampliar seu conhecimento da história de Poços de Caldas, obter noções da criação e modificações ocorridas nos espaços museais, elaborarem metodologias que proporcionem aos alunos 0 entendimento da importância do museu de nossa cidade e incluí-lo, não como expectador, mas como parte integrante de nossa história.

- Abordagens: "Histórica"

- Breve Histórico de Poços de Caldas

- Histórico do surgimento dos museus no Brasil

- Trajetória do Museu Histórico Geográfico de Poços de Caldas

- A História da Villa Junqueira

- A Associação Amigos do Museu - AMIVI

- Sugestões didáticas.

\section{Abordagens do Plano de Ação Cultural 03}

\section{- Plano de Ação Cultural}

- Terceira Abordagem

Ministrante: Haroldo Paes Gessoni

Utilizar os bens culturais como recurso didático, analisar as vivências do passado, entender o momento presente e refletir sobre o futuro.

Durante algum tempo notamos o distanciamento existente entre os programas adotados nas escolas e a realidade dos alunos com relação às atividades extracurriculares. 0 aluno conhecia praticamente tudo sobre História Geral, mas desconhecia a história do seu próprio bairro e da sua cidade.

Atualmente nos deparamos com conteúdos mais flexíveis, programas diversificados, onde os alunos mantêm um contato constante com o Museu e outras instituições, contribuindo consideravelmente no processo educacional, oferecendo novas opções para o trabalho didático e possibi- 
litando um maior aproveitamento, tanto para o Museu, quanto para a escola.

O fato de o Museu Histórico e Geográfico de Poços de Caldas ser uma instituição que realmente representa a cidade, com acervos emergentes da própria comunidade, faz com que o público se identifique muito mais com a realidade local, pois nele está registrada a história de vida do nosso povo e da nossa cidade.

- Abordagens: "Arquitetônicas, Patrimoniais e Culturais"

- Conscientização e Preservação Patrimonial

- Terminologia Patrimonial

- A arquitetura do Complexo Cultural Central

- A arquitetura do complexo Cultural Central

- A Arquitetura dos Prédios tombados em Poços de Caldas, uma proposta para a conscientização e valorização do Patrimônio.

- Sugestões didáticas.
Para o desenvolvimento dessas abordagens criamos uma apostila destinada aos professores a qual está inserida em seu conteúdo, explicações detalhadas do conteúdo do Kit Pedagógico (maquetes). Dessa maneira o professor estará preparado para levar o Kit a escola e trabaIhar de diversas maneiras aplicando uma metodologia diferenciada de acordo com seus objetivos traçados durante 0 ano letivo.

Como propostas finais foram desenvolvidas sugestões didáticas com o objetivo de fixar todo o conteúdo especificado no Kit e plano de ação cultural de uma forma lúdica beneficiando aluno e professor. Esse foi um esboço inicial e uma síntese traçada para o professor com o intuito que crie novas sugestões didáticas, realizando novo efeito multiplicador beneficiando a cultura e as novas gerações.

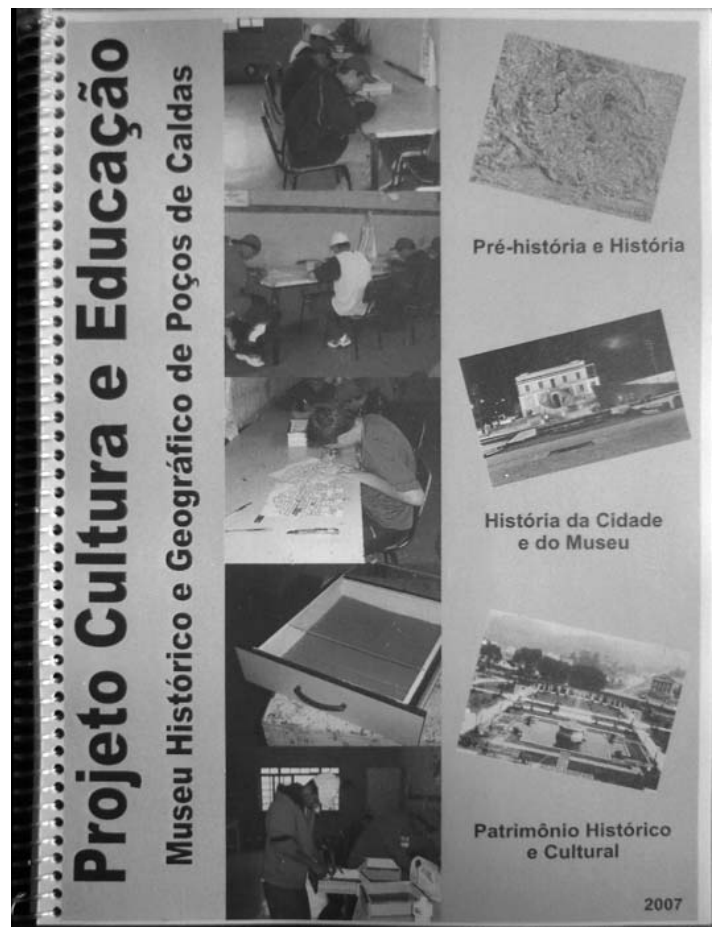

Modelo das Apostilas Confeccionadas 
PROJ ETO CULTURA E EDUCAÇÃO: UMA NOVA PROPOSTA MUSEOLÓGICA REGIONAL NA DIMENSÃO DO MUSEU HISTÓRICO E GEOGRÁFICO DE POÇOS DE CALDAS MG Daniel Fernandes Moreira, Haroldo Paes Gessoni e Sônia Maria Sanches

\section{Esquema dos jogos inseridos nas sugestões didáticas:}

\section{Sugestão didática 01}

\section{LIGUE OS PONTOS}

Desenho esquemático da Fachada do Palace Hotel

Ligue os pontos e descubra a fachada de um dos edifícios tombados pelo CONDEPHACT - Conselho de Defesa do Patrimônio Histórico, Artístico, Cultural e Turístico de Poços de Caldas.

Sugerimos que os professores utilizem uma adaptação podendo abranger qualquer outro edifício.

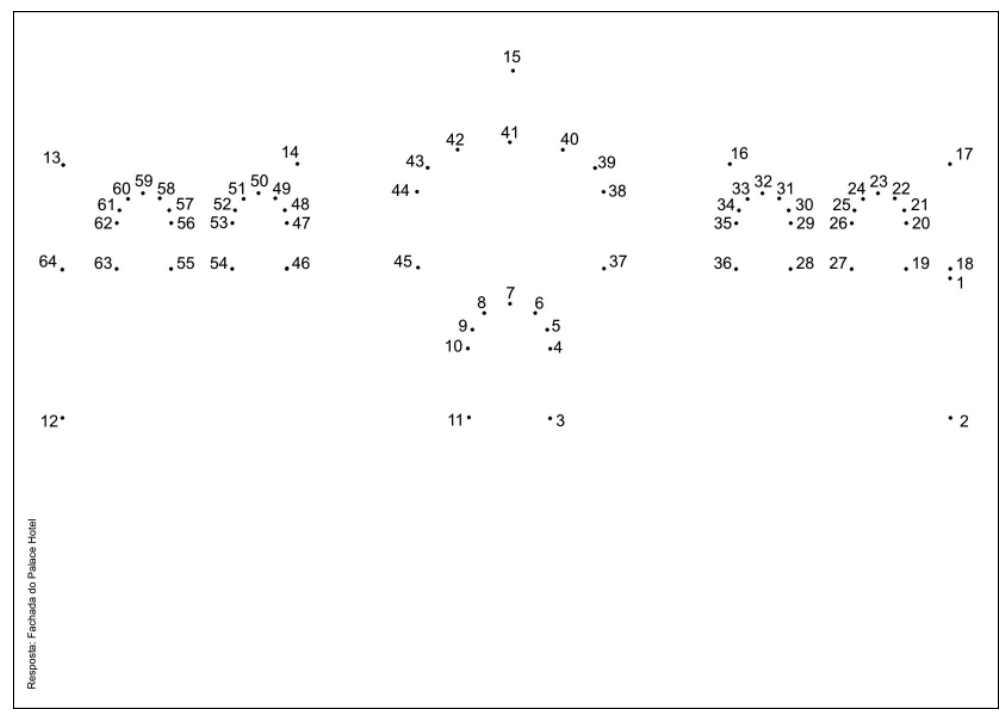

\section{Sugestão Didática 02}

\section{CAÇA-PALAVRAS}

São diversas as formas de proteção do patrimônio cultural, encontre os nomes que significam ações de preservação urbana:
INVENTARIAR

TOMBAMENTO

CONSERVAÇÃO

CONSOLIDAÇÃO

RESTAURAÇÃO

ALORIZAÇÃO

REUTHLIZAÇÃO

\begin{tabular}{|c|c|c|c|c|c|c|c|c|c|c|c|c|c|c|c|c|c|c|c|c|c|c|c|}
\hline $\mathrm{N}$ & U & M & V & 0 & A & $S$ & $\mathrm{~T}$ & $\mathrm{H}$ & $\mathrm{N}$ & $G$ & A & $\mathrm{E}$ & $R$ & $\mathrm{~N}$ & A & $\mathrm{G}$ & $\mathrm{R}$ & Z & $x$ & C & V & $\mathrm{T}$ & $\mathrm{N}$ \\
\hline Z & W & $F$ & C & $\mathrm{s}$ & $D$ & $\mathrm{~F}$ & $\mathrm{G}$ & $x$ & $S$ & W & $E$ & $R$ & $\mathrm{G}$ & $\mathrm{V}$ & $P$ & $\mathbf{R}$ & $A$ & $\mathrm{~S}$ & $D$ & $\mathrm{~F}$ & G & 0 & $\mathrm{~J}$ \\
\hline$x$ & $\mathrm{~S}$ & G & 0 & $E$ & $\mathrm{~N}$ & $E$ & $u$ & $\mathrm{~V}$ & G & Q & $\mathrm{T}$ & 0 & $\mathrm{~S}$ & $\mathrm{~A}$ & 0 & $\mathrm{E}$ & $\mathrm{S}$ & A & $Q$ & $U$ & $E$ & $\mathrm{M}$ & $\mathrm{T}$ \\
\hline C & A & 1 & $\mathrm{~N}$ & $\mathrm{~V}$ & $\mathrm{E}$ & $\mathrm{N}$ & $\mathrm{T}$ & A & $\mathrm{R}$ & I & A & $R$ & $Q$ & $\mathrm{~W}$ & 1 & $\mathbf{U}$ & $S$ & $\mathrm{~T}$ & 0 & $x$ & 1 & $\mathrm{~B}$ & $A$ \\
\hline$Q$ & W & E & $S$ & $\mathrm{R}$ & $T$ & $Y$ & $\mathrm{U}$ & $\mathrm{L}$ & 1 & $\mathrm{R}$ & $\mathrm{K}$ & $\mathrm{L}$ & Ç & $E$ & U & $\mathbf{T}$ & $E$ & $P$ & $\mathrm{~K}$ & 0 & $Q$ & $\tilde{A}$ & $\mathrm{~S}$ \\
\hline $\mathrm{V}$ & $D$ & G & $E$ & $E$ & $U$ & $E$ & 1 & 0 & $\mathrm{~F}$ & $\mathrm{E}$ & $\mathrm{N}$ & G & $\mathrm{H}$ & $\mathrm{D}$ & $Y$ & 1 & $R$ & 0 & $\mathrm{~J}$ & $C$ & W & $\mathrm{M}$ & $D$ \\
\hline B & G & $\mathrm{H}$ & $R$ & $\mathrm{~s}$ & $R$ & $D$ & 0 & $R$ & $D$ & 1 & $B$ & $\mathrm{~F}$ & G & $x$ & $\mathrm{~T}$ & $\mathbf{L}$ & $\mathrm{T}$ & 1 & $\mathrm{H}$ & B & $\mathrm{E}$ & $E$ & $\mathrm{~F}$ \\
\hline $\mathrm{N}$ & $\mathrm{F}$ & $\mathrm{J}$ & V & $\mathrm{E}$ & Z & $S$ & $E$ & 1 & $S$ & C & 0 & $\mathrm{~N}$ & $\mathrm{~s}$ & 0 & L & I & $D$ & A & Ç & A & 0 & $\mathrm{~N}$ & G \\
\hline$M$ & $\mathrm{H}$ & $\mathrm{K}$ & $A$ & $w$ & $x$ & $A$ & $\mathrm{R}$ & Z & A & $\mathrm{E}$ & $\mathrm{V}$ & $D$ & $\mathrm{~F}$ & $z$ & $R$ & $\mathbf{z}$ & $Y$ & $Y$ & $\mathrm{G}$ & $\mathrm{F}$ & $\mathrm{T}$ & $\mathrm{T}$ & $\mathrm{H}$ \\
\hline B & A & $\mathrm{N}$ & Ç & $\mathrm{R}$ & $E$ & $\mathrm{~S}$ & $\mathrm{~T}$ & A & $U$ & $\mathrm{R}$ & A & Ç & $\tilde{A}$ & 0 & $E$ & A & $U$ & $\mathrm{~T}$ & $\mathrm{~F}$ & $E$ & $\mathrm{Y}$ & 0 & $\mathrm{~J}$ \\
\hline A & $\mathrm{E}$ & L & $\tilde{A}$ & 1 & C & $D$ & $\mathrm{C}$ & Ç & $\mathrm{N}$ & $\mathrm{A}$ & C & $\mathrm{S}$ & $\mathrm{s}$ & $\mathrm{E}$ & W & Ç & 1 & A & $\mathrm{D}$ & $Y$ & $\mathrm{U}$ & $\mathrm{C}$ & $\mathrm{K}$ \\
\hline$S$ & $\mathrm{~T}$ & Ç & 0 & $\mathrm{U}$ & G & $A$ & $\mathrm{~s}$ & $\tilde{A}$ & $M$ & $\mathrm{R}$ & $x$ & A & $x$ & $\mathrm{R}$ & Q & $\tilde{\mathbf{A}}$ & 0 & $\mathrm{R}$ & $S$ & $\mathrm{~T}$ & 1 & $x$ & $\mathrm{~L}$ \\
\hline$D$ & $Y$ & Q & W & $\mathrm{E}$ & $R$ & $\mathrm{~T}$ & $\mathrm{Y}$ & 0 & $\mathrm{R}$ & 1 & A & L & A & A & U & 0 & $\mathrm{P}$ & 0 & $A$ & 0 & $P$ & $z$ & Ç \\
\hline
\end{tabular}




\section{Sugestão Didática 03}

\section{RECORTE E COLE}

Recortar as fotos dos bens tombados pelo município e relacionar com os temas, colando nos respectivos espaços.
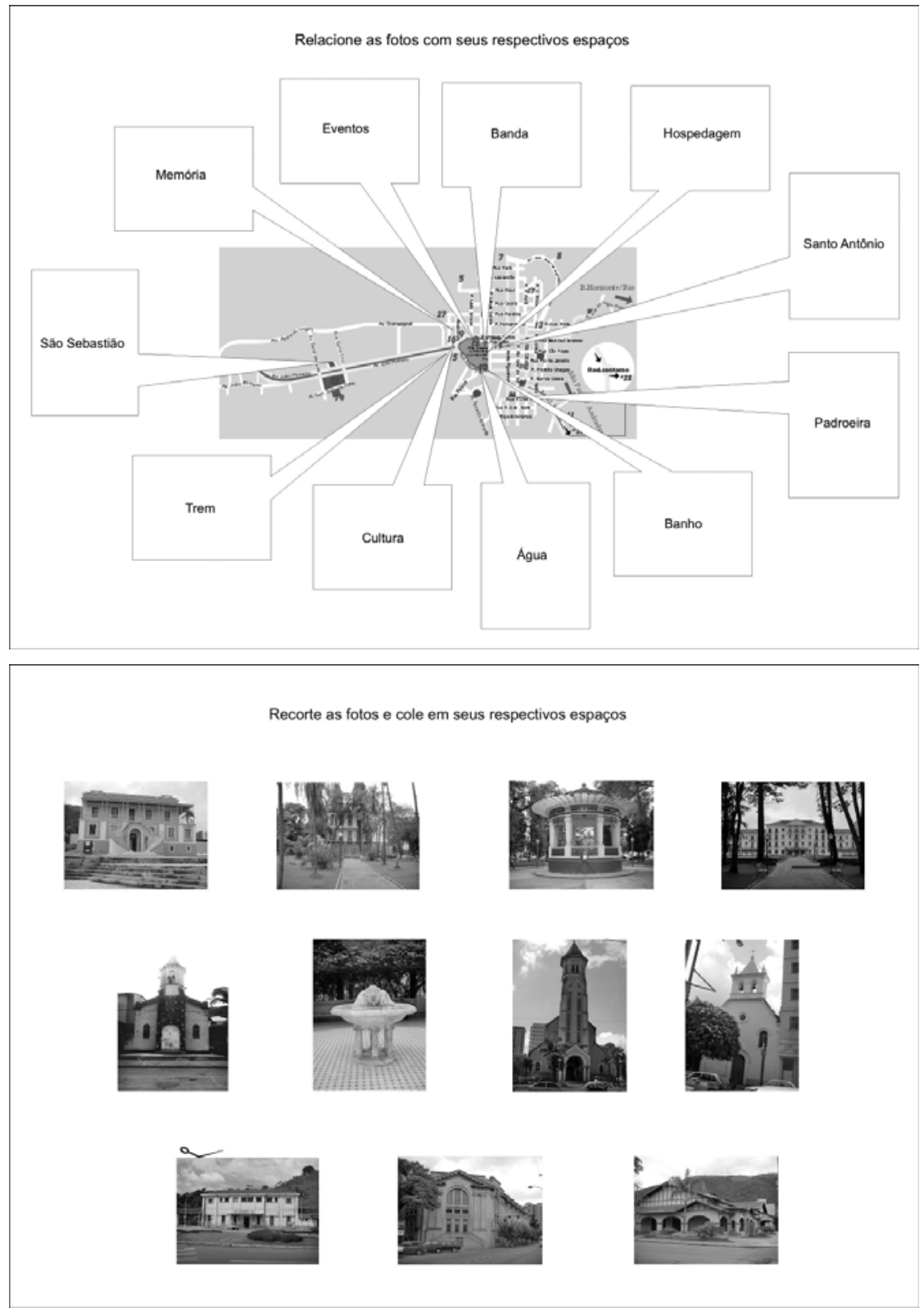
PROJ ETO CULTURA E EDUCAÇÃO: UMA NOVA PROPOSTA MUSEOLÓGICA REGIONAL NA DIMENSÃO DO MUSEU HISTÓRICO E GEOGRÁFICO DE POÇOS DE CALDAS MG Daniel Fernandes Moreira, Haroldo Paes Gessoni e Sônia Maria Sanches

\section{Sugestão Didática 04}

\section{J OGO DA MEMÓRI A}

Consiste em cartas com fotos dos prefeitos da cidade de Poços de Caldas MG, que atualmente são nomes em espaços públicos. As cartas deverão ser colocadas lado a lado
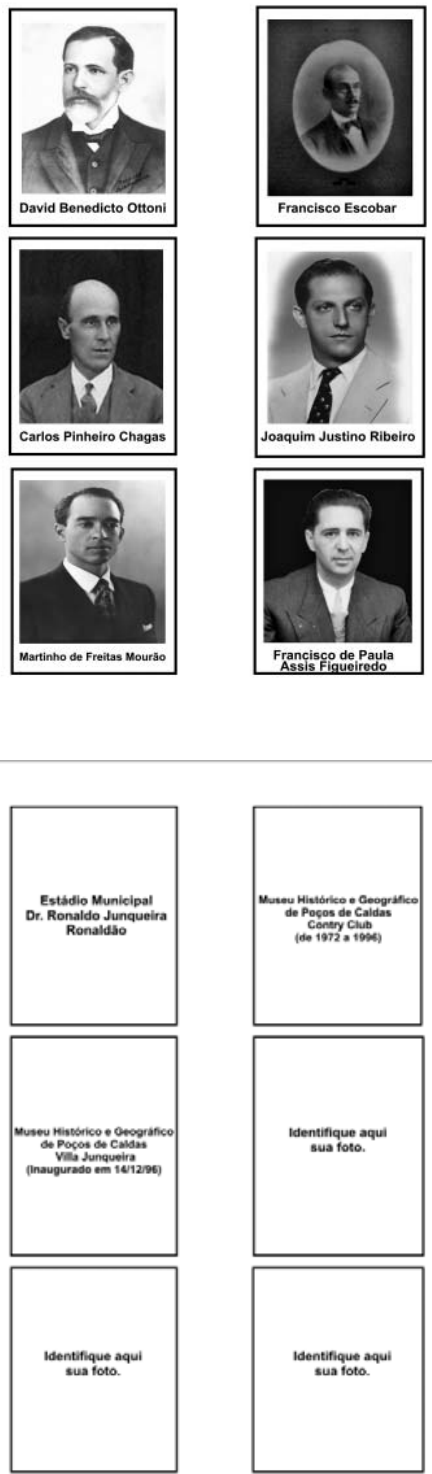

com a frente voltada para baixo. 0 jogador desvira duas cartas. A cada par feito, o jogador retira as cartas. Ganha o jogo quem fizer maior número de pares.

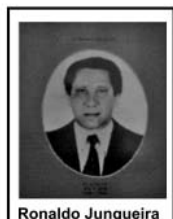

Ronaldo Junqueira
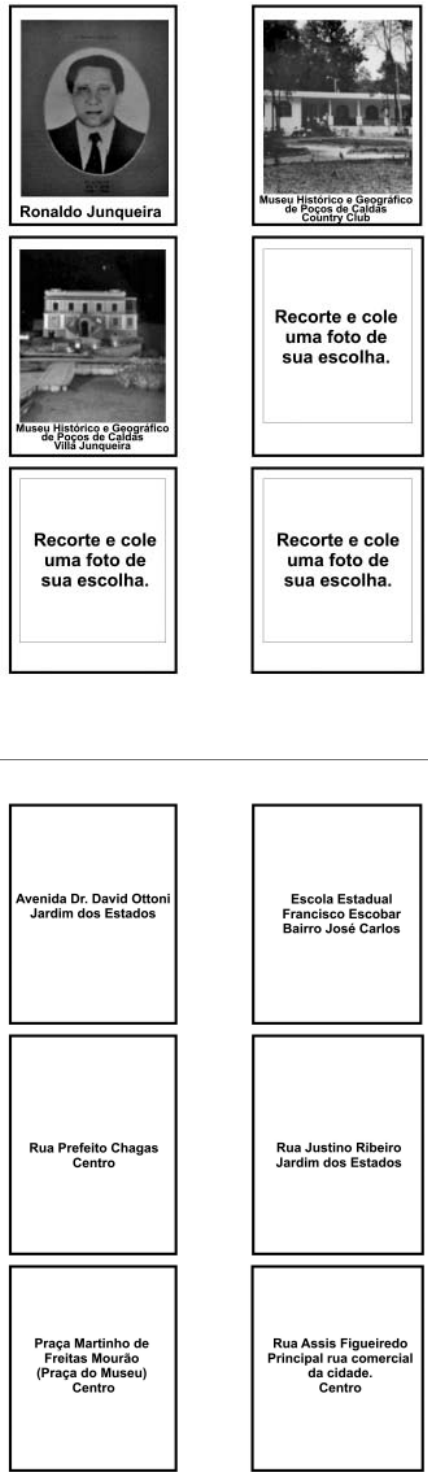


\section{Sugestão Didática 05}

\section{ANTEPASSADO DOS OBJ ETOS}

O aluno (a) deverá observar atentamente as imagens de objetos antigos, depois recortar e colar no lugar indicado o mesmo objeto moderno. Para essa atividade sugerimos os folhetos de propaganda distribuídos em lojas.
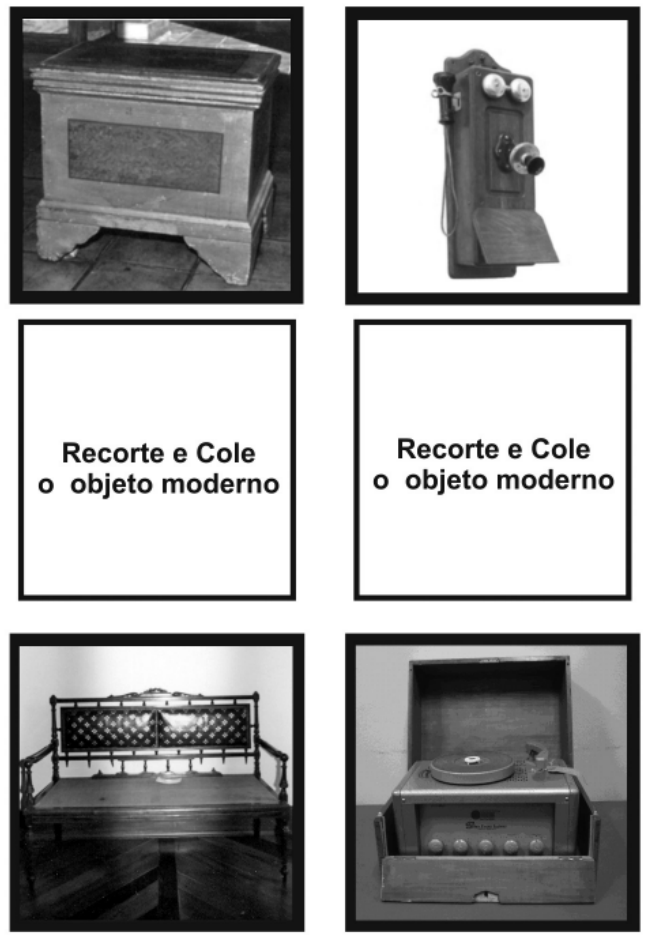

Recorte e Cole o objeto moderno
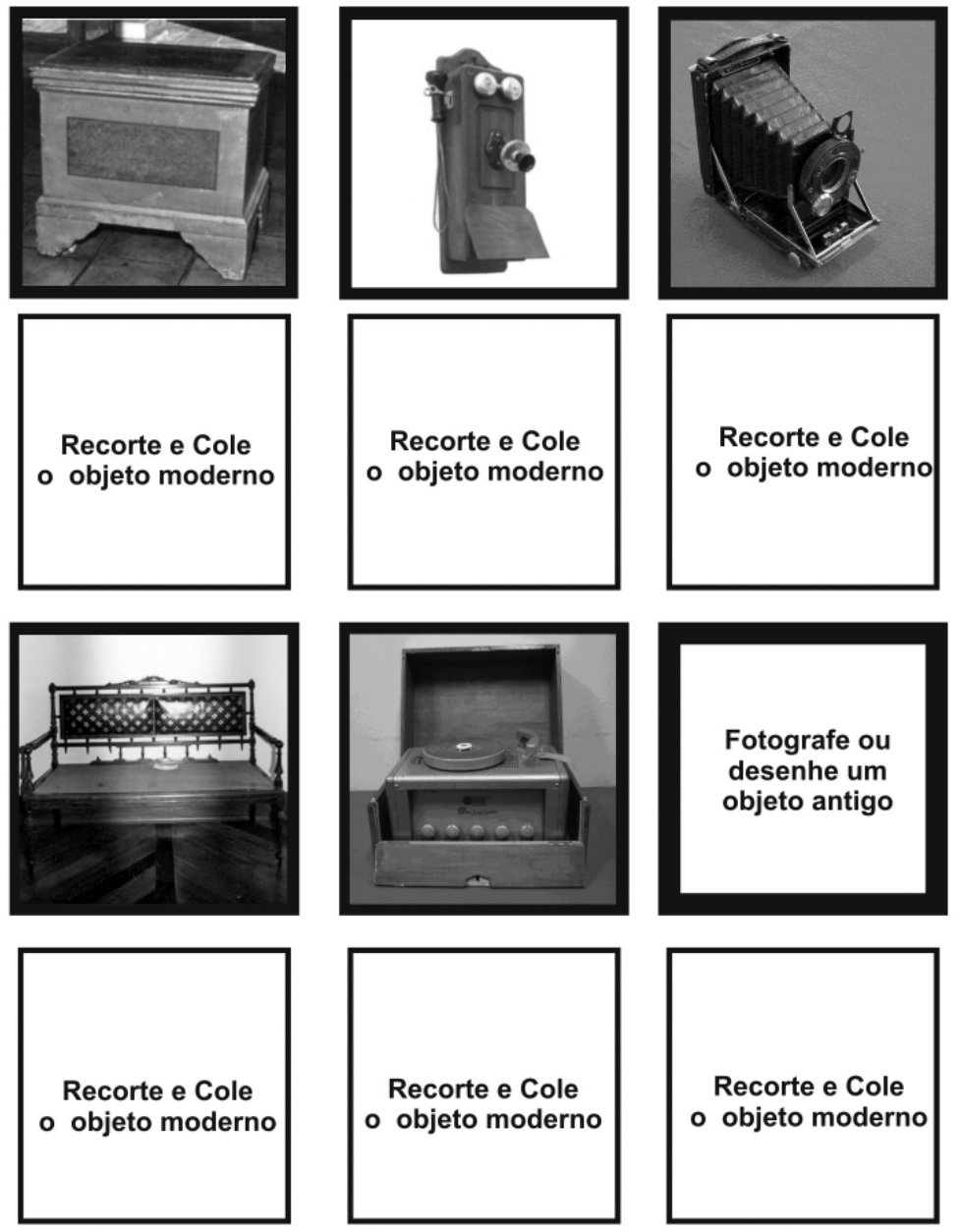
PROJ ETO CULTURA E EDUCAÇÃO: UMA NOVA PROPOSTA MUSEOLÓGICA REGIONAL NA DIMENSÃO DO MUSEU HISTÓRICO E GEOGRÁFICO DE POÇOS DE CALDAS MG Daniel Fernandes Moreira, Haroldo Paes Gessoni e Sônia Maria Sanches

\section{Sugestão Didática 06}

\section{COMPLETE A FACHADA DO MUSEU}

Exercício de observação para desenvolvimento da ação motora e identificação de figuras geométricas.
Os espaços faltantes são completados por desenhos que o aluno fará após observar a mesma figura completa.

OBSERVE A FACHADA DO MUSEU

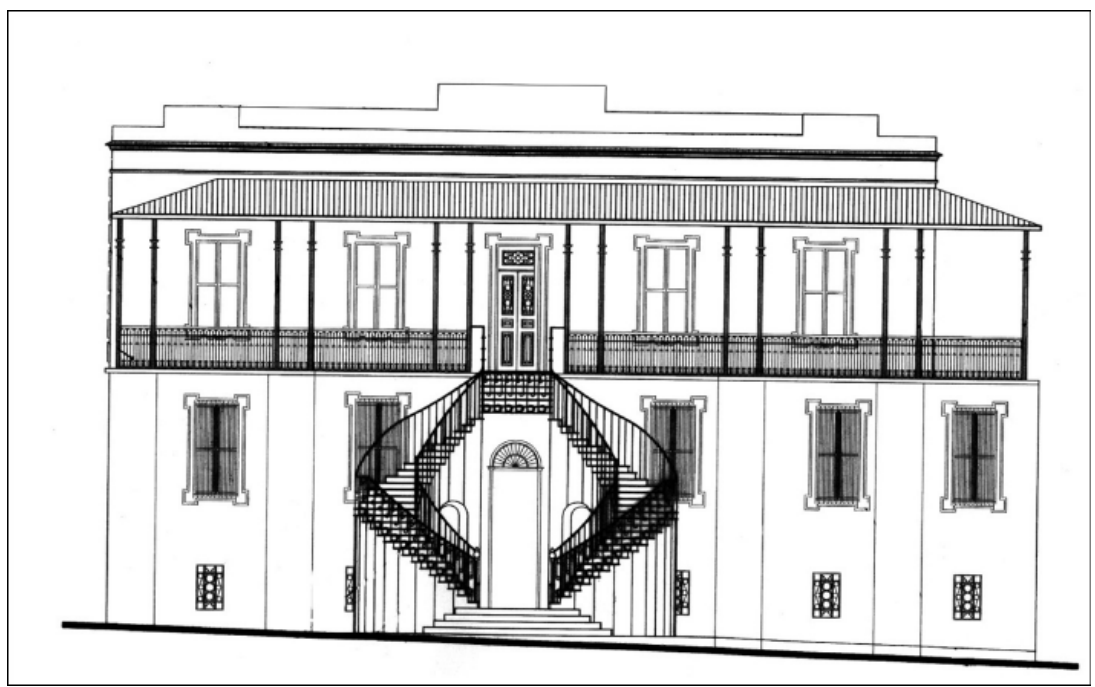

COMPLETE O DESENHO DE ACORDO COM O ANTERIOR.

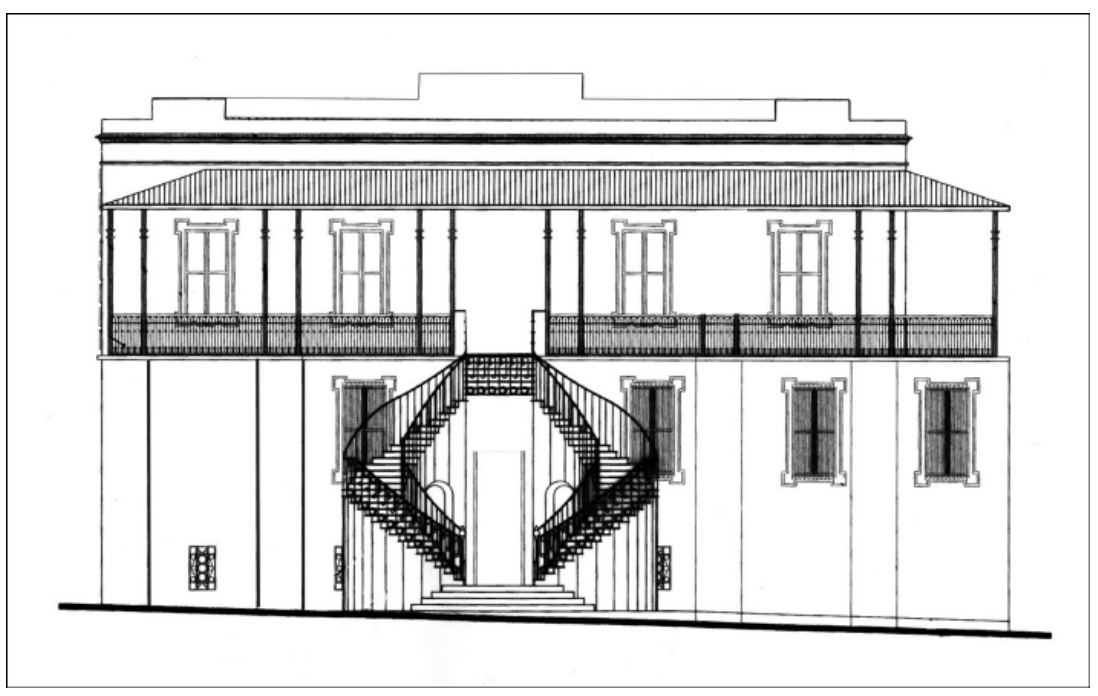




\section{CONCLUSÃO}

O projeto Cultura e Educação proporcionou intercâmbio Cultural entre múltiplas áreas resgatando o valor da cultura em suas mais variadas expressões.

\section{AGRADECIMENTOS}

Ao programa do curso de Pós-Graduação oferecido pelo Museu de Arqueologia e Etnologia e ao serviço Técnico de Musealização - divisão de difusão Cultural.

A Lei Estadual de Incentivo a Cultura do Governo do Estado de Minas Gerais.

A empresa incentivadora Togni S.A Materiais Refratários.

A empresa colaboradora CBA (Companhia Brasileira de Alumínio) - Votorantim.

A Prefeitura Municipal de Poços de Caldas MG.

A Secretaria de Educação de Poços de Caldas MG.

A AMIV - Associação Amigos do Museu.

Ao Museu Histórico e Geográfico de Poços de Caldas.

Dr. Daniel Atencio - Universidade de São Paulo USP.

Dr. Oscar Seguel. Universidade Austral do Chile.
Dra. Márcia Angelina Alves. Universidade de São Paulo USP.

Dr. Astolfo G Araújo. Universidade de São Paulo USP.

Drâ. Erika M.R. González. Universidade de São Paulo USP e Núcleo de Estudos Estratégicos UNICAMP.

Dr. Pedro Paulo Funari. Universidade de São Paulo USP e Núcleo de Estudos Estratégicos UNI CAMP.

Dr. Tomas D. Dillehay. Universidade de Vanderbilt EUA.

MS-Gerson Levi Méndes. Universidade de Vanderbilt EUA.

MS- Carlos Rovaron. Universidade de São Paulo. USP.

MS- Raul Ortiz. Universidade de Campinas UNICAMP.

MS - Gilmar Pinheiro Henrique. Universidade de São Paulo USP.

Ednelson Pereira de Souza (CCPY) - Comissão Pró Yanomami, Universidade Fedreral de Roraima.

Aos geólogos de Poços de Caldas: Dr. Resk Frahya e Dr. Don Williams.

Ivan Moura Antunes. Poços de Caldas PMJ (Plano Municipal da Juventude)

Alunos do PMJ (Plano Municipal da Juventude).

Aos professores e alunos de Caldas MG.

\begin{abstract}
The Project "Culture and Education: a new proposed regional museum in the size of the Museum of History and Geography of Wells Caldas MG" (Certificate 1619/001/2004) was developed in 2007 supported by State Law for Encouragement of Culture no. 12733/97, Decree No. 13665/2000. 43615/2003. It is a project of artistic and cultural character that aims awareness of students and teachers from public schools for the pre historic heritage, historical, geological, architectural, landscape and ecosystem, through a Cultural Action for teachers and making Kits Artistic travelers.
\end{abstract}

Keywords: Culture - prehistory - geology - history - heritage. 


\section{REFERENCI AS BI BLI OGRÁFICAS}

\section{Ação Cultural 01}

ALVES, M. A.

19917 Culturas Ceramistas de São Paulo e Minas Gerais: Um estudo técnico-tipológico. Revista do Museu de Arqueologia e Etnologia $n^{\circ} 1$, p.71-96.

ARAÚJ O, A. G. M \& NEVES. W \& PILÓ L.

2003 Eventos de seca durante o Holoceno no Brasil: Possíveis explicações para o entendimento da variabilidade cultural no período paleoíndio (11.000-8.000 A.P.). CD - Anais do XII Congresso da Sociedade Brasileira de Arqueologia, (Arqueologia das Américas), realizado em São Paulo.

ATENCIO D. \& COUTINHO J, \& ULBRICH M. N. C.

1999 Hainite from Poços de Caldas, Minas Gerais, Brazil. The Canadian Mineralogist. Vol 37, pp. 91-98.

BRUNO, MARIA CRISTINA OLIVEIRA, VASCONCELLOS, CAMILO DE MELLO

1989 A proposta educativa do Museu de PréHistória Paulo Duarte. Revista de Pré-História, São Paulo, n.7, p.161-182.

DIAS JR.

1971 "Breves notas a respeito das pesquisas do PRONAPA no Sul de Minas Gerais". In: Programa Nacional de Pesquisas. Resultados preliminares do quarto ano. 196869. Publ. Avulsas Museu Emílio Goeldi, Belém, 15: 133-138,il.

FUNARI, P. PAULO \& FOGOLARI. E

2005 Estudos de Arqueologia Histórica; Erechim RS.

FUJIMORI K.

1974 Minerais Radioativos do Campo Agostinho, Poços de Caldas (MG). Tese Livre Doc. Inst. Geociências. Universidade de São Paulo.

GUARNIERI, W R.C.

1990 Conceito de cultura e sua inter-relação com o patrimônio cultural e preservação. Cadernos Museológicos, Rio de Janeiro, n.3, p.7-12.

GONZALEZ, E. M. R.

2000 Arqueologia em Perspectiva: 150 anos de prática e reflexão no estudo do nosso passado. W. Neves (org.) Dossiê Antes de Cabral EDUSP.

GORSKY V. A. E GORSKY E.

1974 Contribuição à Mineralogia e Petrografia do Planalto de Poços de Caldas. Companhia Nacional Energia Nuclear. Rio de Janeiro: vol.13,93 p.
INSTITUTO MOREIRA SALLES

1990 Memórias de Poços de Caldas. Poços de Caldas: Casa da Cultura. 23p.

INSTITUTO DO PATRIMÔNIO HISTÓRICO E ARTÍSTICO NACIONAL

Cadastro Nacional de Sítios Arqueológicos. Site: www.iphan.gov.br.

MEGALE, NILZA BOTELHO

1990 Memórias Históricas de Poços de Caldas. Prefeitura Municipal de Poços de Caldas. 235p.

MENDES-SILVA

2006 Caçadores-coletores na Serra de Paranapiacaba durante a transição do holoceno médio para o tardio (5920-1000 anos AP). São Paulo - MAE-USP - Dissertação de Mestrado.

CURT NIMUENDAJU

1987 Mapa etnohistórico - Fundação Instituto Brasileiro de Geografia e Estatística em colaboração com a Fundação Nacional PróMemória. Rio de Janeiro: IBGE.

MORAIS, J. L.

2000 Tópicos de Arqueologia da Paisagem. Revista do Museu de Arqueologia. São Paulo, 10:3-30.

MORAIS, J. L.

1999 A Arqueologia e o Fator Geo. Rev. do Museu de Arqueologia e Etnologia, São Paulo, 9: 3-22.

MOURÃO, B. M.

1992 Medicina Hidrológica - Moderna Terapêutica das Águas Minerais e Estâncias de Cura. Ed. Prima.

PONTES, HUGO

2004 A Poesia das Águas: Retratos escritos de Poços de Caldas. Poços de Caldas: Ed. Sulminas.

PROUS, A.

1992 Arqueologia Brasileira, Universidade de Brasília.

RELATÓRIO DE DIAGNÓSTICO DE POTENCIAL AR-

QUEOLÓGICO

2004 Área do decreto DNPM 834.761/93. Mineração Curimbaba Ltda. Município de Poços de Caldas - MG: (MC Consultoria, Ltda).

SAINT-H, A.

1779-1853 Viagem à Província de São Paulo. Universidade de São Paulo. Série Coleção Reconquista de Brasil. 1976.

MARRAS, STÉLIO

2004 A propósito de águas virtuosas e ocorrências de uma estação balneária no Brasil. Belo Horizonte: Editora UFMG.

SLAVEC, B. G \& MANTOVANI. M \& SHUKOWSKY. W. 2004 Estudo Gavimétrico do Maciço Alcalino 
de Poços de Caldas. Revista Brasileira de Geociências, volume 34 (2): 275-280.

SPIX, J. B. VON \& MARTIUS, F. VON

1976 Viagem pelo Brasil. São Paulo: Ed. MeIhoramentos.

WILLIAMS, DON. PRADO, ALEX

2001 Memorial da Companhia Geral de Minas História da Mineração de Poços de Caldas.

\section{Ação Cultural 02}

\section{CADERNO DE DIRETRIZES MUSEOLÓGICAS I \\ 2002 Belo Horizonte: Secretaria de Estado da Cultura/Superintendência de Museus. \\ MEGALE, NILZA BOTELHO \\ 2002 Memórias Históricas de Poços de Caldas. Poços de Caldas: Gráfica e Editora Sulminas. \\ PONTES, HUGO \\ 1999110 Anos de Imprensa Poços-caldense. Poços de Caldas: Gráfica e Editora Sulminas.}

\section{Ação Cultural 03}

\section{MOURÃO, MÁRIO}

1951 Poços de Caldas - Síntese Histórico-social. 2a edição. São Paulo, SP: Oficinas Gráficas de Saraiva S.A.

1933 Poços de Caldas - Esboço Histórico, Águas Mineraes Sulfurosas. Rio de Janeiro: Papelaria Velho.

OTTONI, HOMERO BENEDICTO

1960 Poços de Caldas. São Paulo. SP: Editora Anhambi. Oficinas de Artes Gráficas Bisordi S.A.

FRANCO, GABRIEL JUNQUEIRA; JUNQUEIRA, LUIZ ALBERTO FRANCO

1983 Família Franco - Genealogia e História. Poços de Caldas, MG: Gráfica D. Bosco.

MOURÃO, BENEDICTUS MÁRIO

1998 Quarteto Construtor de Poços de Caldas e epopéia de Pedro Sanches. Poços de Caldas, MG: Gráfica Sulminas.
ULBRICH H. H. E GOMES C. B.

1981 Alkaline rocks from continental Brazil. Earth Science Rev. 17, 135-154.

ULBRICH H. H. ULBRICH M. N. C.

1992 O Maciço Alcalino MG-SP - Características Petrográficas e Estruturais. Roteiro de Excursão do 370 Congresso Brasileiro de Geologia, SBG/SP Vol. 5 - São Paulo-SP.

2006 Artigo sobre a criação da Associação Amigos do Museu.

SANTOS, FAUSTO HENRIQUE DOS

2000 Metodologia Aplicada em Museus. São Paulo: Editora Mackenzie.

SANTOS, MARIA C. T. M.

1987 Museu, Escola e Comunidade - Uma integração necessária. Ministério da Cultura. Sistema Nacional de Museus. Salvador.

PIMENTA, REYNALDO DE OLIVEIRA

1998 O Povoamento do Planalto da Pedra Branca - Caldas e Região. Obra póstuma: Reynaldo de Oliveira Pimenta. Complemento: Colcha

SEGUSO, MÁRIO de Retalhos/Marta Amato. São Paulo: s. ed.

1999 Os Admiráveis Italianos de Poços de Caldas - 1884-1915. Poços de Caldas, MG: Gráfica D. Bosco.

PRADO, ALEX

Memorial da Companhia Geral de Minas. História da Mineração no Planalto de Poços de Caldas. Poços de Caldas, MG: Santa Edwiges Artes Gráficas, Tietê, SP: 2001.

MEGALE, NILZA BOTELHO

2002 Memórias Históricas de Poços de Caldas. 2a edição revisada e ampliada. Poços de Caldas, MG: Gráfica Sulminas. 